\title{
Prostate Cancer Survivorship and Supportive Care: Guidance Needed
}

\author{
Sanchia S. Goonewardene ${ }^{1 *}$, Raj Persad ${ }^{2}$ and A. Young ${ }^{3}$ \\ ${ }^{1}$ Spr/ Mphil Student, University of Warwick, Guys Hospital, Kings College, UK \\ ${ }^{2}$ Warwick University, $U K$ \\ ${ }^{3}$ Bristol Southmead Hospital, UK
}

Received: 19 December, 2015; Accepted: 12 May, 2016; Published: 12 May, 2016

\section{Dear Sir,}

Survivorship is a growing area of cancer care and is infrequently addressed. Whilst there is guidance on PSA interpretation and of 'acute' care, there are currently no specific guidelines addressing 'holistic' care for this cohort, including their medical comorbidity. The definition of survivor is from a diagnosis of cancer onwards (National Coalition of Cancer Survivors, 2013) which can be extended to include those receiving palliative therapy, e.g. androgen deprivation therapy. Due to the health workloads in today's current clinical practice, we seem more inclined to 'treat' the PSA and less likely to look at the issues the patient has and address them. A failing on our part, which needs to change.

Questions regarding the physical implications of cancer survivorship have been highlighted by the Institute of Medicine, 2006 (Institute of Medicine, 2006). These unmet needs are a significant burden on the healthcare system (National Cancer Survivorship Initiative, Department of Health, 2008). Each of the papers published address cardiovascular health, stroke and diabetes in this survivorship population on androgen deprivation therapy. This still remains unaddressed in the younger survivorship population, who have undergone radical therapy. This is further contributed to by the fact men with prostate cancer are more likely to die of cardiovascular disease. So the question is what can we do to address them?

Good survivorship care needs good insight into the patients' requirements, not just medically, but holistically. If an alternative method of follow-up is to be developed, there are some core principles which must be considered. Firstly, good communication between patients and health care professionals. To allow this, patients should have a personalised survivorship care plan, including assessment of medical comorbidities. This allows focused assessment and treatment, in the survivorship phase (Ferrell B.R. et al, 1998).

An information prescription should be part of that, to ensure patients remain well informed. Giving survivors information about disease and treatment aids self-management. It also enables them to become more self-aware about their own health. Any survivorship programme, should allow the healthcare team to identify disease recurrence early.

Simple interventions such as GP educational events as part of a survivorship programme can support this. We investigated GPs' views of our previously published programme. We detected low confidence levels with managing prostate cancer and side effects of treatment. Half of the GPs we surveyed were not fully informed about the survivorship programme: 25\% thought it was a programme to empower patients who are cured, and $15 \%$ thought it was a programme with a holistic approach, with nothing else offered. However, after the educational intervention, these were corrected. This goes to show what a useful component of a survivorship team primary care can be especially with addressing chronic ongoing medical problems.

In conclusion, to further the development of prostate cancer survivorship and supportive care, guidelines, taking into account medical comorbidities are needed. 